\title{
Colloidal quantum dot based solar cells: from materials to devices
}

\author{
Jung Hoon Song ${ }^{1}$ and Sohee Jeong ${ }^{1,2^{*}}$
}

\begin{abstract}
Colloidal quantum dots (CQDs) have attracted attention as a next-generation of photovoltaics (PVs) capable of a tunable band gap and low-cost solution process. Understanding and controlling the surface of CQDs lead to the significant development in the performance of CQD PVs. Here we review recent progress in the realization of low-cost, efficient lead chalcogenide CQD PVs based on the surface investigation of CQDs. We focus on improving the electrical properties and air stability of the CQD achieved by material approaches and growing the power conversion efficiency (PCE) of the CQD PV obtained by structural approaches. Finally, we summarize the manners to improve the PCE of CQD PVs through optical design. The various issues mentioned in this review may provide insight into the commercialization of CQD PVs in the near future.
\end{abstract}

Keywords: Colloidal quantum dots, Nanocrystals, Solar cells, Photovoltaics, Lead chalcogenides

\section{Introduction}

Colloidal quantum dots (CQDs) are chemically-prepared semiconductor nanocrystals, which have diameter is less than twice the Bohr radius describing the spatial extension of exciton (electron-hole pair) in semiconductors. The CQDs have attracted attention over the past decade due to a solution based synthetic methods [1], easily tunable optoelectronic properties [2], and superior processing capabilities for optoelectronic applications [3]. Specifically, lead chalcogenide CQDs are considered as a prominent material for a next-generation photovoltaics (PVs) [4] owing to their wide tunable bandgaps covering from visible to near-infrared wavelength regime arising from a large Bohr exciton radius and narrow bulk bandgap. Also, the excitons in lead chalcogenide CQDs can be easily separated into electrons and holes because of their high dielectric constant and extinction coefficient. More importantly, because their material properties can be easily controlled using electron density functional design, lead chalcogenide CQDs are expected to exhibit efficient utilization of low-energy and low-intensity photons and

\footnotetext{
*Correspondence: sjeong@kimm.re.kr

${ }^{1}$ Nano-Convergence Systems Research Division, Korea Institute

of Machinery and Materials (KIMM), Daejeon 34113, Republic of Korea

Full list of author information is available at the end of the article
}

efficient collection of high-energy charges, which are difficult to achieve in conventional PVs [5]. In this article, we discuss the current state of high-efficiency CQD PV development.

\section{Size-dependent physical properties of CQDs}

When the size of a bulk semiconductor is reduced, discrete energy levels appear in the energy band owing to the quantum confinement effect, as shown in Fig. 1a. A CQD consists of a semiconductor core and surface ligands. In the case of a core, synthesis methods capable of controlling the size of binary or ternary compound semiconductors, such as II-VI (CdSe, CdS) compound semiconductors, III-V (InP, InAs), IV-VI (PbS, PbSe), and III-V $\left(\mathrm{CuInS}_{2}, \mathrm{CuInSe}_{2}\right)$, have been developed and reported. As shown in Fig. 1a, the bandgap of IV-VI ( $\mathrm{PbS}, \mathrm{PbSe}$ ) CQDs can be controlled to absorb light in the range of $600-3000 \mathrm{~nm}$, which is suitable for solar cell materials. Additionally, most of the reported high-efficiency CQD PVs have been fabricated using IV-VI (PbS, $\mathrm{PbSe}$ ) CQDs. Therefore, in this article, we mainly focus on the IV-VI (PbS, PbSe) CQDs. Typically, when the energy bandgap of a semiconductor decreases in a singlejunction $\mathrm{PV}$, the open-circuit voltage $\left(\mathrm{V}_{\mathrm{OC}}\right)$ decreases and the short-circuit current $\left(\mathrm{J}_{\mathrm{SC}}\right)$ increases by absorbing more light. Figure $1 \mathrm{~b}$ shows that single-junction CQD 

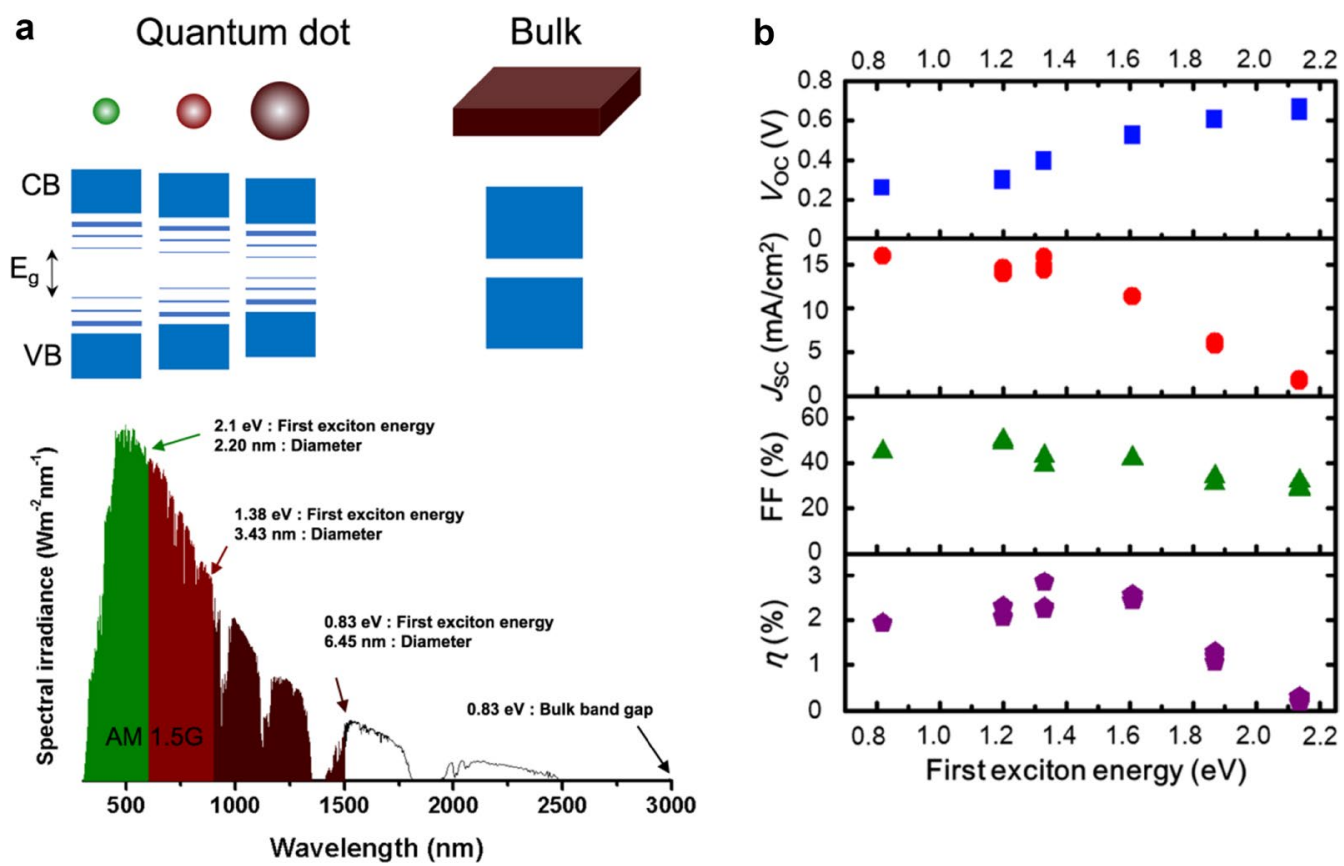

Fig. 1 a AM 1.5G solar spectrum (from ASTM G173-03 reference spectra), band diagram, and first exciton energy of PbS CQDs with various diameters. b Characteristics of Schottky junction PbS CQD PVs as a function of the first exciton energy (reprinted with permission from ref. 6, Copyright 2013 American Physical Society)

PVs also exhibit the relationship between energy bandgaps and solar cell characteristics as described above [6]. In CQD solids, the hole mobility increases depending on the size of the CQDs [7], explained by the decrease in the total number of interparticle hops and the reduction of the coulombic charging energy of an individual particle.

\section{Surface modification and characteristics control}

Nanomaterials such as CQDs generally have a high surface-area-to-volume ratio and the surfaces are prone to form dangling bonds, which causes defects. Especially, reducing surface defects is very important for obtaining high-efficiency CQD PVs. A CQD fabricated using wet-chemical synthesis consists of a semiconductor core and surface ligands, as shown in Fig. 2a [8]. The physical characteristics of the CQD can be controlled by the surface ligands owing to its high surface-area-to-volume ratio. Surface ligands generally have an amphiphilic structure consisting of a polar head group and a nonpolar aliphatic group. The rear portion of the ligand, which is composed of aliphatic groups, provides steric stabilization and dispersibility, which is the ability to dissolve in organic solvents. The head group typically contains amines, carboxylate, thiolate and phosphonate. These functional groups bind to the cationic metals on the CQD surfaces and produce nonstoichiometric CQDs, resulting in a doping effect. Typically, compound semiconductors exhibit p-type doping polarity under anion-rich conditions and n-type doping polarity under cation-rich conditions. Thus, doping can be controlled by using these ligands. When a CQD film is fabricated, the mobility of the charge carriers is determined by the length of ligands that passivate the surface of the CQDs. As shown in Fig. 2b, the mobility and coupling of the CQDs increase with the use of shorter-length ligands [9]. In addition, when surface ligands bind to the surface of CQDs, the electron distribution of the CQD surface and ligand changes, resulting in the formation of dipole moment on the CQD surface. The strength and direction of the surface dipole moment are determined by the surface ligands. Therefore, the position of the energy band is shifted by the surface dipole moment, as shown in Fig. 2c-d [10]. CQDs have many surface defects because of their high surface-area-to-volume ratio. Therefore, the surface ligands play an important role in reducing these surface defects. Figure $2 \mathrm{e}-\mathrm{f}$ show that additional passivation using halide atoms reduces the surface defects of CQDs and increases photoluminescence (PL) [11, 12]. Furthermore, as passivation prevents the oxidation of CQDs, their air stability can be improved $[11,12]$. Consequently, the surface ligands of CQDs play an important role in controlling the dispersibility, air stability, and electrical properties (such as doping, mobility, electronic structure, and surface defects). Therefore, understanding 
a

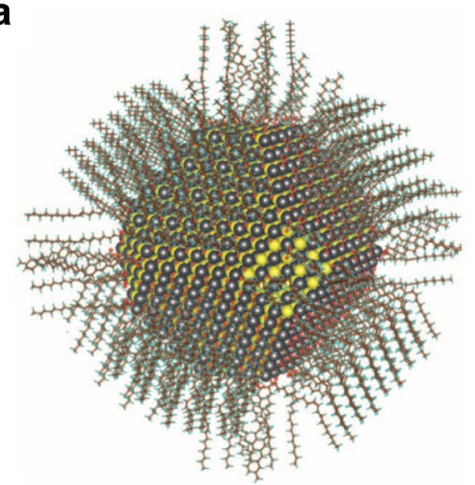

C
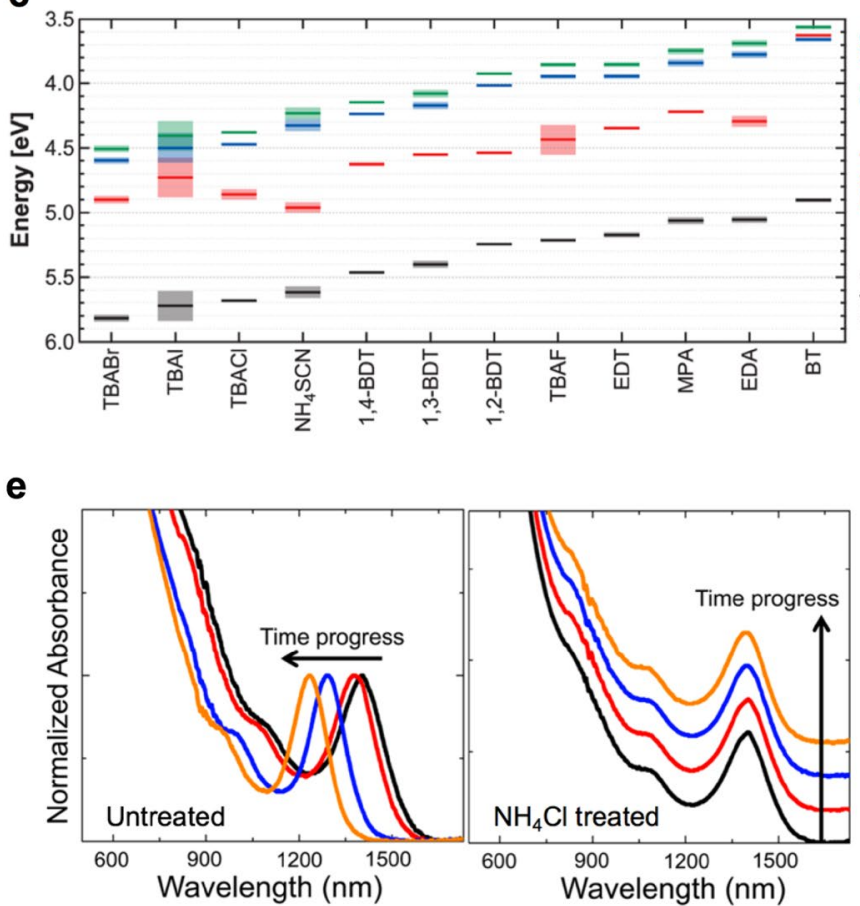

b
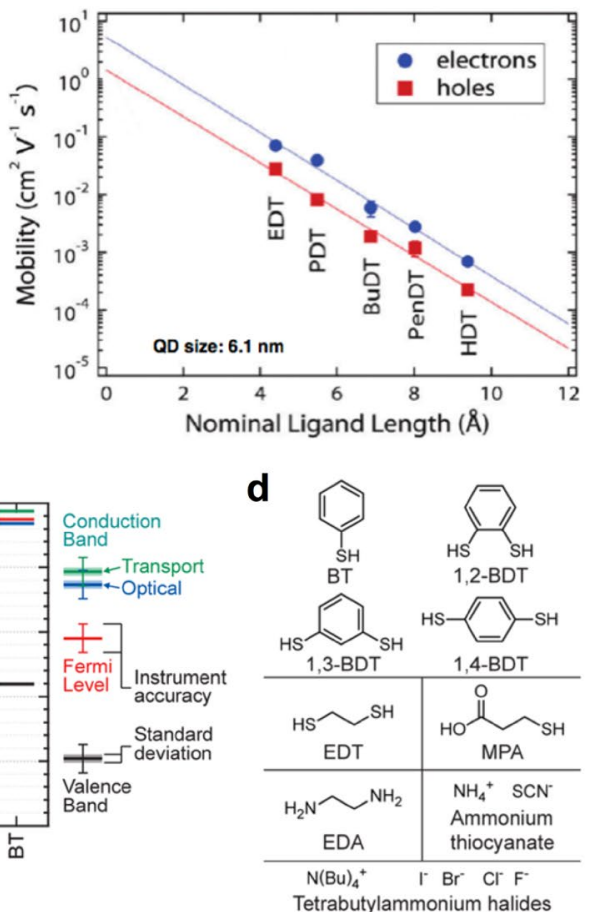

d

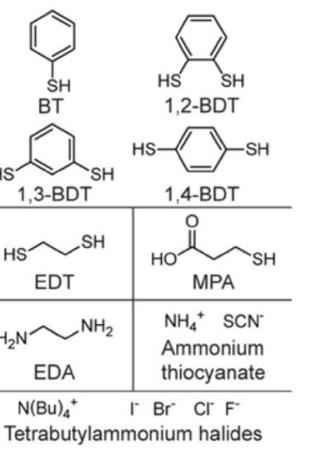

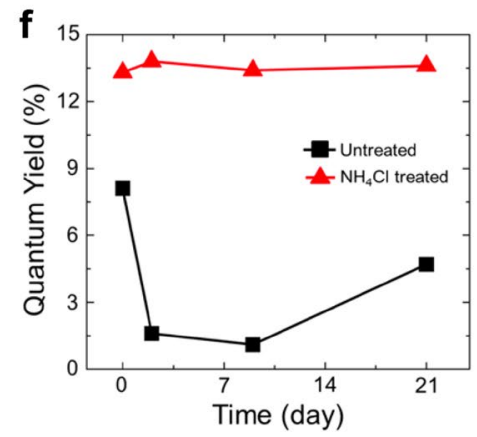

Fig. 2 a Schematic diagram of a PbS CQD consisting of a semiconductor core and surface ligands (reprinted with permission from ref. 8, Copyright 2014 American Association for the Advancement of Science). b Charge carrier mobility of PbS CQD films with various surface ligand length (reprinted with permission from ref. 9, Copyright 2013 American Chemical Society). c Energy band position of PbS CQD films for d different surface ligands (reprinted with permission from ref. 10, Copyright 2013 American Chemical Society). Air stability analysis using e the absorbance spectrum and $\mathbf{f}$ quantum yield of $\mathrm{PL}$ as a function of storage time and its dependence on $\mathrm{NH}_{4} \mathrm{Cl}$ treatment on the surface of PbSe CQDs (reprinted with permission from ref. 11, Copyright 2014 American Chemical Society)

the dependence of CQD characteristics on surface ligands is essential for obtaining high-efficiency CQD PVs.

\section{Enhancement of power conversion efficiency through solar cell structure design}

The power conversion efficiency (PCE) of CQD PVs has increased very rapidly since national renewable energy laboratory (NREL) certification began in 2010 and the highest PCE in the NREL chart is currently $13.4 \%$. In the early stages of the development of CQD PVs, the PCE was increased in accordance with the structural changes of the devices. However, since 2012, the development of technologies that control the surface of CQDs has resulted in dramatic improvements of the performance of CQD PVs. In Sect. 4, we will describe the structural development of CQD PVs. Initially, CQDs with high extinction coefficients were used instead of dyes in dyesensitized solar cells. In this case, the CQD absorbs light to form excitons, and electrons and holes separated 
from the excitons are generally transferred through $\mathrm{TiO}_{2}$ and the electrolyte, respectively (Fig. 3a) [13]. Consequently, the electric characteristics of the CQDs have relatively low influence on the device in the dye-sensitized solar cell. However, the CQDs with uncontrolled electrical properties can be formed as a monolayer on $\mathrm{TiO}_{2}$ to absorb only a small amount of light. Therefore, mesoporous $\mathrm{TiO}_{2}$ is commonly used to overcome this problem. In recent years, new CQDs without heavy metals (such as $\mathrm{Zn}-\mathrm{Cu}-\mathrm{In}-\mathrm{Se} \mathrm{CQDs}$ ) have been employed to produce a dye-sensitized solar cell structure that exhibits a relatively high PCE [14-16].

Currently, most of the high-efficiency CQD PVs use a thin film solar cell structure. For the PbS CQD solar cells, the excitons generated by light are easily separated by the internal field of the diode due to their high dielectric constant, and the separated electrons and holes move in the CQD thin film. Therefore, their electronic properties itself largely influence on the CQD solar cells. Such electronic properties can be controlled by chemically surface treatment. Initially, thin-film CQD PVs used a Schottky diode structure with a metal (Fig. 3b) [13, 17]. However, the QD film thickness cannot be increased, originated by the small built-in potential and narrow space charge region (SCR) in such Schottky junction CQD PVs. In addition, their charge carrier diffusion length is very short (several tens of nanometers) due to many surface trap states. For the high-efficiency CQD PVs, the SCR

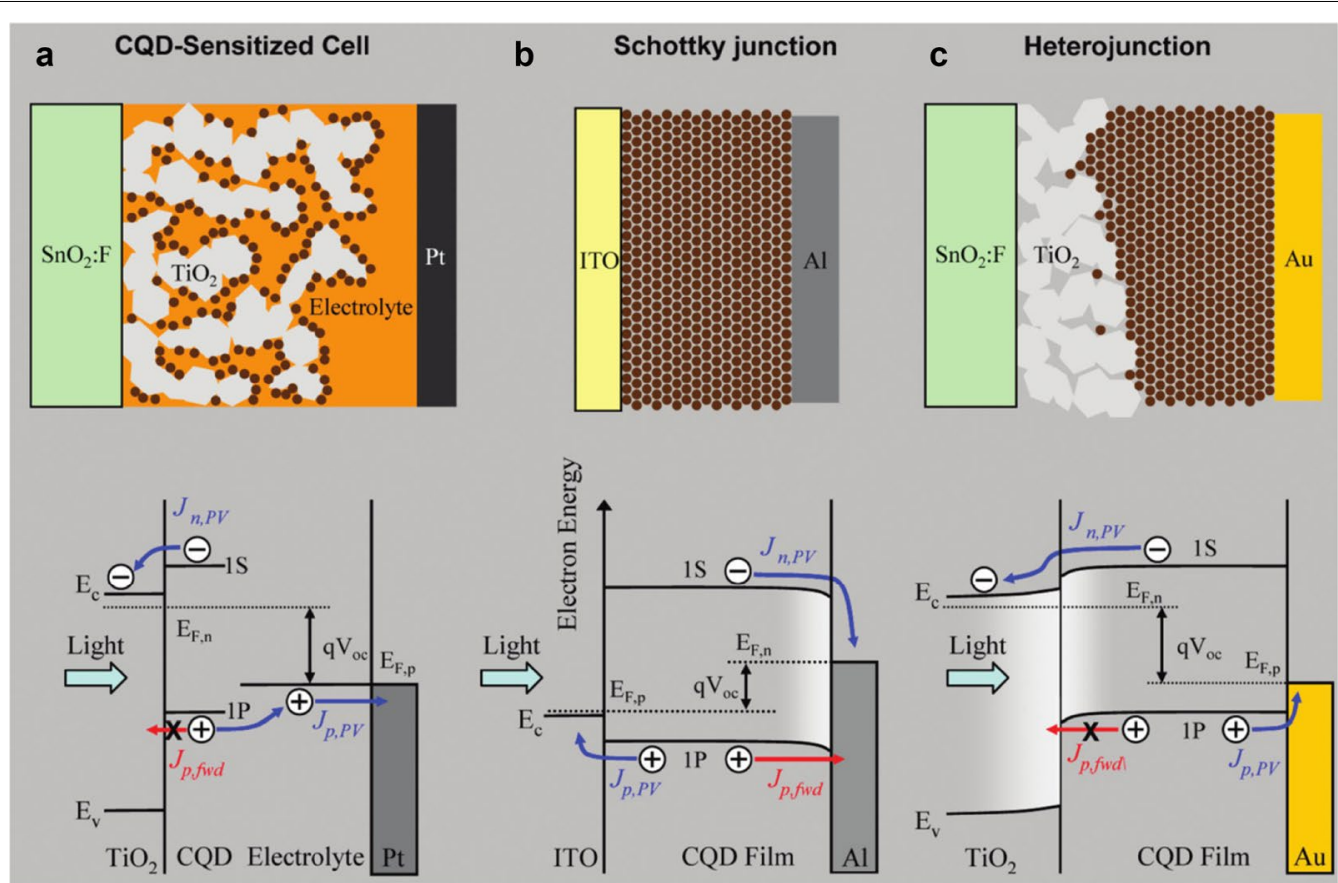

d Quantum junction
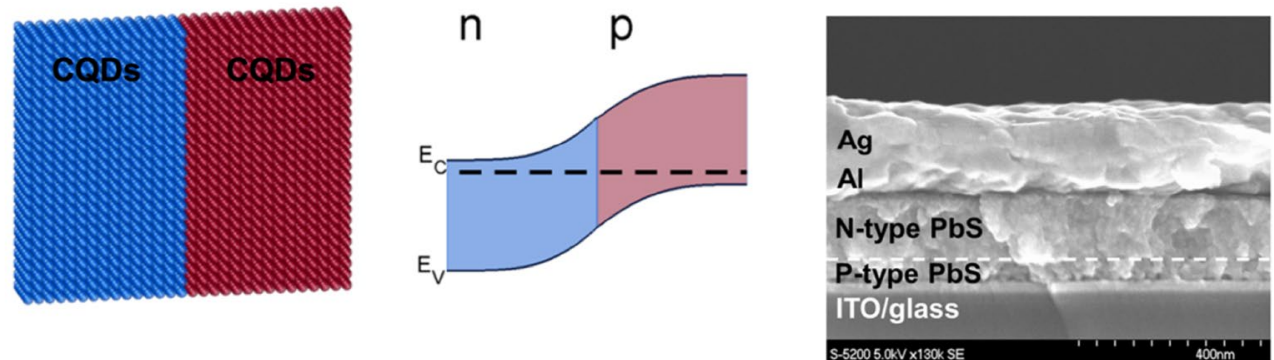

Fig. 3 Schematic of photovoltaic architectures and flat-band diagrams at $V_{O C}$ of a CQD-sensitized solar cell, b Schottky junction solar cell, and $\mathbf{c}$ heterojunction solar cell (reprinted with permission from ref. [13], Copyright 2010 American Chemical Society). d Schematic of CQD homojunction solar cell, energy band diagram, and cross-sectional image of scanning electron microscope image (reprinted with permission from ref. [19], Copyright 2012 American Chemical Society) 
should be enlarged. To overcome this problem, heterojunction diodes have been fabricated using oxide semiconductors with n-type doping polarity such as $\mathrm{ZnO}$ and $\mathrm{TiO}_{2}$ (Fig. 3c) [13, 18].

Finally, a homojunction CQD PV with CQDs of n-type and p-type doping polarities has been successfully developed by stoichiometry control using surface ligands (Fig. 3d). The structure of the homojunction shows a lower efficiency than heterojunction structures. In a heterojunction using oxide semiconductors with relatively large energy bandgaps, such as $\mathrm{ZnO}$ and $\mathrm{TiO}_{2}$, the electrons move easily at the interface between the CQD and the oxide semiconductor; however, the holes cannot move across the interface and the recombination of electrons and holes decreases. Therefore, the homojunction CQD PVs show lower PCE than heterojunction ones [19].

\section{Enhancement of power conversion efficiency through surface modification}

The surface modification of a CQD is an important factor that determines not only the characteristics of the CQD but also the characteristics of the resulting PV device. Figure 4 shows the efficiencies of CQD PVs, as reported by the NREL chart. As shown in the efficiency chart, there are significant developments of the CQD PVs efficiencies over the last 7 years as understanding and controlling the surface of CQDs. In the case of CQDs

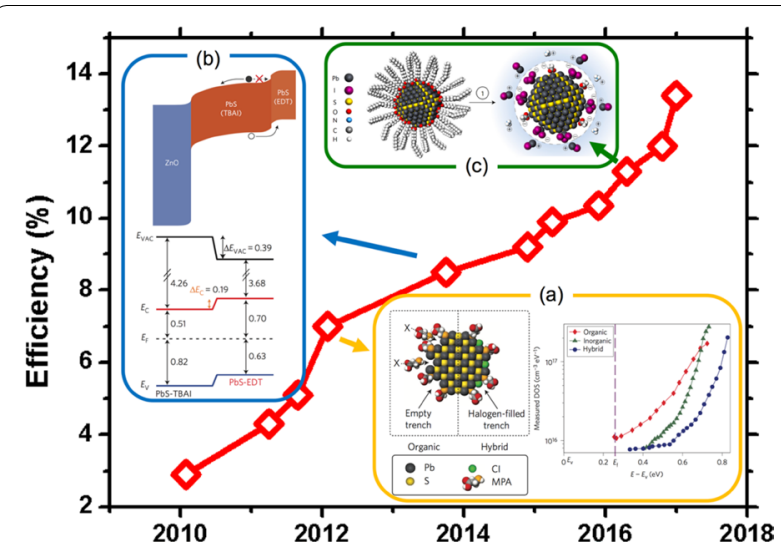

Fig. 4 NREL efficiency chart of CQD PVs. a Schematic of a CQD treated with an MPA ligand and a chloride (inorganic) ligand; density of states in the energy bandgap obtained from a transient photovoltage measurement (reprinted with permission from ref. 21, Copyright 2012 Macmillan Publishers Ltd.). 6 Band diagram of a CQD PV based on electron blocking with EDT-exchanged CQDs; energy band positions for various ligands obtained from ultraviolet photoelectron spectroscopy analysis (reprinted with permission from ref. 22, Copyright 2014 Macmillan Publishers Ltd.). c Schematic of CQD and CQD ink (reprinted with permission from ref. 25, Copyright 2016 Macmillan Publishers Ltd.) fabricated by wet-chemical synthesis, the surface is passivated by long organic ligands to reduce internal defects and improve size uniformity due to high synthesis temperatures. To fabricate a thin-film CQD PV, long organic ligands must be replaced with short ligands to improve carrier mobility. When forming a conductive CQD thin film, a layer-by-layer (LBL) process is used to repeatedly perform the deposition of the CQD film and the ligand exchange process to reduce cracks. After the synthesis, $\mathrm{PbS}$ CQDs consist of Pb-rich nonstoichiometric (111) surfaces passivated by ligands and stoichiometric (100) surfaces without ligands [20]. The long organic ligands on the non-stoichiometric (111) surface are replaced by mercaptopropionic acid (MPA) ligands containing sulfur (anion), leading to the p-type behavior in CQDs. Thus a heterojunction CQD PV with the p-type of CQDs and n-type of $\mathrm{TiO}_{2}$ or $\mathrm{ZnO}$ can be fabricated [21]. As mentioned in Sect. 4, the CQDs have many surface defects and a very short diffusion length; and thus charge carriers are mainly driven by the drift in the electric field inside the SCR. Therefore, to achieve high-efficiency CQD PVs, the CQD active layer should increase without decreasing the extraction efficiency of photo-induced carriers through improving the carrier diffusion length. The (100) surface has no surface ligands and is easily oxidized to generate defects. When producing CQD PVs with halidetreated CQDs (Fig. 2e-f), both the air stability can be improved and the efficiency can be increased by improving carrier diffusion length due to reduction of surface defects (Fig. 4a) [21].

In addition, the electron blocking layer (EBL) at the interface between the CQD thin film and the metal electrode is used in the same principle to prevent recombination by blocking the hole transport at the interface. In this case, EBL prevents the movement of electrons to the metal electrode through the energy band shift of ethanedithiol (EDT)-treated CQD film [22]. Therefore, highefficiency CQD PVs can be achieved by using an EBL to reduce electron recombination. In this structure, the number of surface defects and the type of ligands vary depending on the acidity of the protonic solvent used in the ligand exchange process. An efficiency higher than $10 \%$ has been reported for CQD PVs produced by changing the electrical properties of CQD films with various protic solvents in the ligand exchange process (Fig. 4b) [23].

The fabrication of CQD PVs is based on the LBL process by employing the above ligand exchange process. This fabrication procedure is time-consuming and the electrical properties of the CQD film are modified by the environment during the ligand exchange process. Therefore, the LBL process reduces the reproducibility of CQD PVs and inhibits their commercialization. To solve this 
problem, the surface of $\mathrm{PbS} C \mathrm{CQDs}$ has been completely modified with ammonium iodide $\left(\mathrm{NH}_{4} \mathrm{I}\right)$, which has the closest affinity with $\mathrm{Pb}$, to allow the CQDs to be dissolved in $\mathrm{N}, \mathrm{N}$-dimethylformamide [24]. Using a technique similar to the above method and considering the dependence of the energy band shift on the ligand, CQDs with a PCE of $11.3 \%$ have been obtained (Fig. 4c) [25]. Ligand exchange performed in the liquid phase is more complete than solid ligand exchange because it increases the diffusion length by suppressing surface defects, resulting in increased thickness of the CQD film without reducing the efficiency.

\section{Enhancement of power conversion efficiency using optical design}

In CQDs, the quantum confinement effect separates the levels of the energy band of bulk semiconductors, resulting in an increase in the energy bandgap and a discrete energy level, as shown in Fig. 1a. This discrete energy level decreases the density of state and reduces the amount of absorbed light around the energy bandgap. Therefore, observations of the external quantum efficiency (EQE) spectrum of a CQD PV show a markedly decreased efficiency near the energy bandgap. This is a unique characteristic of the CQD. To solve this problem,
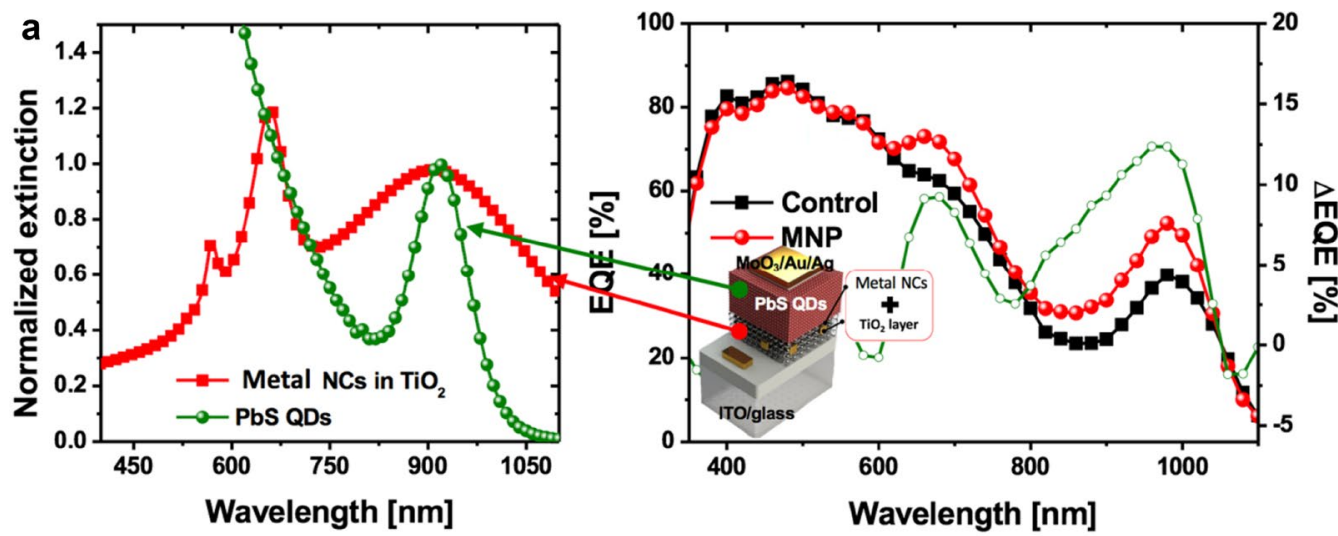

b
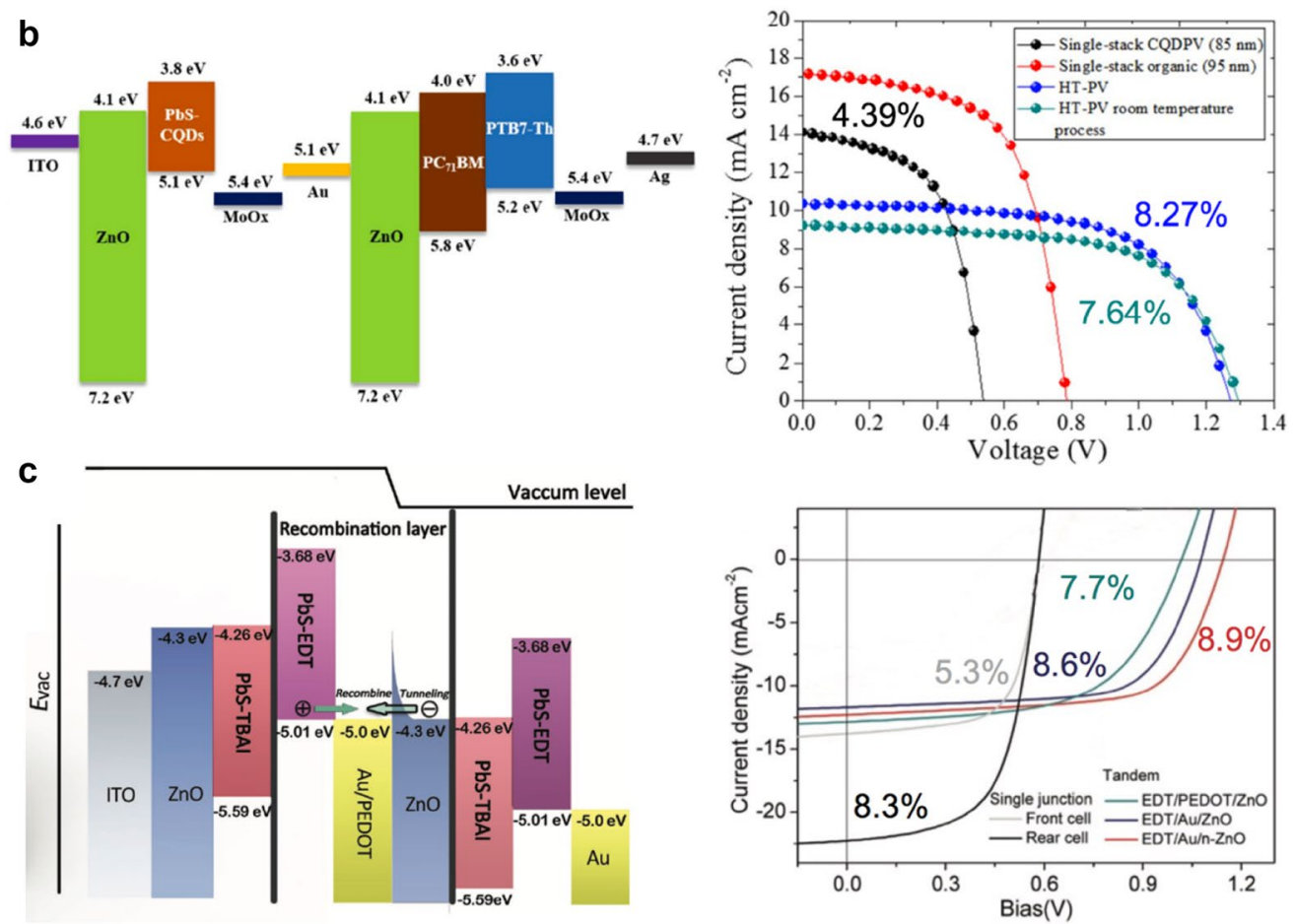

Fig. 5 a Absorption spectra of metal nanoparticles in $\mathrm{TiO}_{2}$ and PbS CQDs; EQE spectra with and without metal nanoparticles (reprinted with permission from ref. 26, Copyright 2015 Wiley-VCH Verlag GmbH \& Co. KGaA). b Energy band diagram and J-V curves of multi-junction PVs using an organic PV and a CQD PV (reprinted with permission from ref. 27, Copyright 2016 Elsevier B.V.). c Energy band diagram and J-V curves of multijunction PVs using CQD PVs (reprinted with permission from ref. 28, Copyright 2017 Wiley-VCH Verlag GmbH \& Co. KGaA) 
surface plasmons of metal nanoparticles have been used to shift the spectrum of the incident sunlight to the region in which CQDs can absorb large amounts of light, thereby improving the PCE (Fig. 5a) [26].

Multiple junctions using CQDs improve the PCE because they provide easier control of the light absorption region of the CQDs, thus adjusting the energy bandgap. Although relatively few studies have been conducted on multi-junction CQD PVs, they have shown a possibility of maximized efficiency. The LBL process is performed to fabricate the conductive CQD film. In this case, because an organic ligand has a specific acidity and LBL process is performed using a polar solvent and a nonpolar solvent alternately, the pre-formed front subcell and intermediate recombination layer (RL) is damaged and the efficiency is lowered. Therefore, as shown in Fig. 5b, a CQD PV with a smaller bandgap than that of the organic PV is placed in the front sub-cell, thereby minimizing the reduction in efficiency [27]. In addition, intermediate RLs have been developed that can induce sufficient electron and hole recombination without damage by using the solution process during the formation of the rear sub-cells to minimize the efficiency reduction (Fig. 5c) [28]. However, the current efficiency of multijunction CQD PVs is lower than that of single-junction CQD PVs; therefore, further research is required to address these problems. One possible solution is to minimize the damage of the front sub-cell by using CQDs in which the ligand is exchanged in the liquid phase mentioned in the previous section.

\section{Conclusions}

CQDs have been attracting much attention because they can absorb light above their energy bandgap with a high extinction coefficient and can be processed by using a solution process. Significant progress has been achieved in the development of CQD PVs by understanding their surface characteristics and using surface modification to obtain superior characteristics that distinguish them from general bulk semiconductors. In the future, we aim to address the various issues presented in this article and to commercialize CQD PVs along with the commercialization of CQD display fields.

\section{Authors' contributions}

JS and SJ wrote the manuscript. Both authors read and approved the final manuscript.

\footnotetext{
Author details

${ }^{1}$ Nano-Convergence Systems Research Division, Korea Institute of Machinery and Materials (KIMM), Daejeon 34113, Republic of Korea. ${ }^{2}$ Department of Nanomechatronics, University of Science and Technology (UST), Daejeon 34113, Republic of Korea.
}

\section{Acknowledgements}

Not applicable.
Competing interests

The authors declare that they have no competing interests.

Availability of data and materials

Not applicable.

Ethics approval and consent for publication

The authors approve publication of this material.

\section{Funding}

This work was supported by the Global Frontier R\&D program of the Center for Multiscale Energy Systems (NRF-2017M3A6A7051087), NRF program (2016R1A2B3014182), the Global R\&D program (1415134409) funded by KIAT, and the Basic Research Fund from KIMM.

\section{Publisher's Note}

Springer Nature remains neutral with regard to jurisdictional claims in published maps and institutional affiliations.

Received: 21 June 2017 Accepted: 28 July 2017

Published online: 07 August 2017

References

1. M.A. Hines, G.D. Scholes, Colloidal PbS nanocrystals with size-tunable near-infrared emission: observation of post-synthesis self-narrowing of the particle size distribution. Adv. Mater. 15, 1844-1849 (2003)

2. L. Li, P. Reiss, One-pot synthesis of highly luminescent InP/ZnS nanocrystals without precursor injection. J. Am. Chem. Soc. 130, 11588-11589 (2008)

3. J. Jang, H.C. Shim, Y. Ju, J.H. Song, H. An, J.-S. Yu, S.-W. Kwak, T.-M. Lee, I. Kim, S. Jeong, All-solution-processed PbS quantum dot solar modules. Nanoscale 7, 8829-8834 (2015)

4. G.I. Koleilat, L. Levina, H. Shukla, S.H. Myrskog, S. Hinds, A.G. PattantyusAbraham, Sargent. Efficient, stable infrared photovoltaics based on solution-cast colloidal quantum dots, ACS Nano 2, 833-840 (2008)

5. O.E. Semonin, J.M. Luther, S. Choi, H.-Y. Chen, J. Gao, A.J. Nozik, M.C. Beard, Efficiency exceeding 100\% via MEG in a quantum dot solar cell. Sci. 334, 1530-1533 (2011)

6. H. Choi, J.K. Kim, J.H. Song, Y. Kim, S. Jeong, Increased open-circuit voltage in a Schottky device using PbS quantum dots with extreme confinement. Appl. Phys. Lett. 102(19), 193902-1-193902-4 (2013)

7. J. K. Kim, J. H. Song, H. Choi, S. J. Baik, S. Jeong, Space charge limited conduction in ultrathin PbS quantum dot solid diodes. J Appl Phys. 115(5), 054302-1-054302-6 (2014)

8. D. Zherebetskyy, M. Scheele, Y. Zhang, N. Bronstein, C. Thompson, D. Britt, M. Salmeron, P. Alivisatos, L.-W. Wang, Hydroxylation of the surface of PbS nanocrystals passivated with oleic acid. Sci. 344, 1380-1384 (2014)

9. Y. Liu, M. Gibbs, J. Puthussery, S. Gaik, R. Ihly, H.W. Hillhouse, M. Law, Dependence of carrier mobility on nanocrystal size and ligand length in PbSe nanocrystal solids. Nano Lett. 10, 1960-1969 (2010)

10. P.R. Brown, D. Kim, R.R. Lunt, N. Zhao, M.G. Bawendi, J.C. Grossman, V. Bulovic, Energy level modification in lead sulfide quantum dot thin films through ligand exchange. ACS Nano 8, 5863-5872 (2014)

11. J.Y.Woo, J.-H. Ko, J.H. Song, K. Kim, H. Choi, Y.-H. Kim, D.C. Lee, S. Jeong, Ultrastable PbSe nanocrystal quantum dots via in situ formation of atomically thin halide adlayers on PbSe(100). J. Am. Chem. Soc. 136, 8883-8886 (2014)

12. W.K. Bae, J. Joo, L.A. Padilha, J. Won, D.C. Lee, Q. Lin, W.-K. Koh, H. Luo, V.I. Klimov, J.M. Pietryga, Highly effective surface passivation of PbSe quantum dots through reaction with molecular chlorine. J. Am. Chem. Soc. 134, 20160-20168 (2012)

13. A.G. Pattantyus-Abraham, I.J. Kramer, A.R. Barkhouse, X. Wang, G. Konstantatos, R. Debnath, L. Levina, I. Raabe, M.K. Nazeeruddin, M. Grazil, E.H. Sargent, Depleted-heterojunction colloidal quantum dot solar cells. ACS Nano 4, 3374-3380 (2010)

14. M.-K. Mehdi, Z. Zabihullah, S.-N. Masoud, Facile and novel chemical synthesis, characterization, and formation mechanism of copper sulfide 
(Cu2S, Cu2S/CuS, CuS) nanostructures for increasing the efficiency of solar cells. J. Phys. Chem. C 120, 2096-2108 (2016)

15. J.-Y. Kim, J. Yang, J.H. Yu, W. Baek, C.-H. Lee, H.J. Son, T. Hyeon, M.J. Ko, Highly efficient copper-indium-selenide quantum dot solar cells: suppression of carrier recombination by controlled ZnS overlayers. ACS Nano 5, 11286-11295 (2015)

16. J. Du, Z. Du, J.-S. Hu, Z. Pan, Q. Shen, J. Sun, D. Long, H. Dong, L. Sun, X. Zhong, L.-J. Wan, Zn-Cu-In-Se quantum dot solar cells with a certified power conversion efficiency of 11.6\%. J. Am. Chem. Soc. 138, 4201-4209 (2016)

17. J. Tang, X. Wang, L. Brzozowski, D.A.R. Barkhouse, R. Debnath, L. Levina, E.H. Sargent, Schottky quantum dot solar cells stable in air under solar illumination. Adv. Mater. 22, 1398-1402 (2010)

18. J. Gao, C.L. Perkins, J.M. Luther, M.C. Hanna, H.-Y. Chen, O.E. Semonin, A.J. Nozik, R.J. Ellingson, M.C. Beard, n-Type transition metal oxide as a hole extraction layer in PbS quantum dot solar cells. Nano Lett. 11, 3263-3266 (2011)

19. J. Tang, H. Liu, D. Zhitomirsky, S. Hoogland, X. Wang, M. Furukawa, L. Levina, E.H. Sargent, Quantum junction solar cells. Nano Lett. 12, 4889-4894 (2012)

20. H. Choi, J.-H. Ko, Y.-H. Kim, S. Jeong, Steric-hindrance-driven shape transition in PbS quantum dots: understanding size-dependent stability. J. Am. Chem. Soc. 135, 5278-5281 (2013)

21. A.H. Ip, S.M. Thon, S. Hoogland, O. Voznyy, D. Zhitomirsky, R. Debnath, L. Levina, L.R. Rolly, G.H. Carey, A. Fischer, K.W. Kemp, I.J. Kramer, Z. Ning, A.J. Labelle, K.W. Chou, A. Amassian, E.H. Sargent, Hybrid passivated colloidal quantum dot solids. Nat. Nanotechnol. 7, 577-582 (2012)
22. C.-H.M. Chuang, P.R. Brown, V. Bulovic, M.G. Bawendi, Improved performance and stability in quantum dot solar cells through band alignment engineering. Nat. Mater. 13, 796-801 (2014)

23. J.H. Song, H. Choi, Y.-H. Kim, S. Jeong, High performance colloidal quantum dot photovoltaics by controlling protic solvents in ligand exchange. Adv. Energy Mater. 1700301, 1-6 (2017)

24. S. Kim, J. Noh, H. Choi, H. Ha, J.H. Song, H.C. Shim, J. Jang, M.C. Beard, S. Jeong, One-step deposition of photovoltaic layers using iodide terminated PbS quantum dots. J. Phys. Chem. Lett. 5, 4002-4007 (2014)

25. M. Liu, O. Voznyy, R. Sabatini, F.P. Garcia de Arquer, R. Munir, A.H. Balawi, X. Lan, F. Fan, G. Walters, A.R. Kirmani, S. Hoogland, F. Laquai, A. Amassian, E.H. Sargent, Hybrid organic-inorganic inks flatten the energy landscape in colloidal quantum dot solids. Nat. Mater. 1700301, 1-7 (2016)

26. S.-W. Baek, J.H. Song, W. Choi, H. Song, S. Jeong, J.-Y. Lee, A resonanceshifting hybrid $n$-type layer for boosting near-infrared response in highly efficient colloidal quantum dots solar cells. Adv Mater. 27, 8102-8108 (2015)

27. H. Aqoma, R. Azmi, S.-H. Oh, S.-Y. Jang, Solution-processed colloidal quantum dot/organic hybrid tandem photovoltaic devices with $8.3 \%$ efficiency. Nano Energy 31, 403-409 (2017)

28. G. Shi, Y. Wang, Z. Liu, Y. Wang, K. Lu, S. Chen, X. Ling, Y. Li, S. Cheng, W. $\mathrm{Ma}$, Stable and highly efficient $\mathrm{PbS}$ quantum dot tandem solar cells employing a rationally designed recombination layer. Adv. Energy Mater. 1602667, 1-8 (2017)

\section{Submit your manuscript to a SpringerOpen ${ }^{\circ}$ journal and benefit from:}

- Convenient online submission

$\checkmark$ Rigorous peer review

- Open access: articles freely available online

- High visibility within the field

- Retaining the copyright to your article

Submit your next manuscript at $\gg$ springeropen.com 